\title{
Tonotopic organization of the sources of human auditory steady-state responses
}

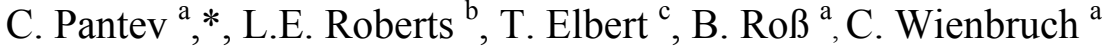 \\ ${ }^{a}$ Center of Biomagnetism, Institute of Experimental Audiology, University of Miinster, Kardinal-von-Galen-Ring 10, D-48129 Munster, Germany \\ ${ }^{b}$ Department of Psychology, McMaster University, Hamilton, Ont. L8S 4K], Canada \\ ${ }^{c}$ Department of Psychology, University of Konstanz, D-78434 Konstanz, Germany
}

\begin{abstract}
Steady-state responses (SSRs) or steady-state fields (SSFs) show maximum amplitude when tone pulses are presented at repetition rates near $40 \mathrm{~Hz}$. This result has led to the hypothesis that the SSR/SSF consists of superimposed transient 'middle latency' responses which display wave periods near $40 \mathrm{~Hz}$ and summate with one another when phase locked by $40 \mathrm{~Hz}$ steady-state stimulation. We evaluated this hypothesis by comparing the cortical sources of the $40 \mathrm{~Hz}$ auditory SSF with sources of the middle latency Pa wave which is prominent in electrical and magnetic recordings, and with the cortical sources of the familiar N1 wave, at different carrier frequencies between 250 and 4000 Hz. SSF sources determined for the different carrier frequencies were found to display a 'medial' tendency tonotopy resembling that of the N1m (sources for the higher frequencies represented more deeply within the supratemporal sulcus), opposite the 'lateral' tendency tonotopy of the middle latency Pam (sources for the higher frequencies situated more laterally). A medial SSF tonotopy was observed in each of the subjects investigated, including three subjects for whom Pam and N1m maps were also available. These findings suggest that the $40 \mathrm{~Hz}$ SSF may not consist of summated or entrained middle latency responses, as has previously been proposed. Alternative mechanisms for the SSR are discussed.
\end{abstract}

Keywords: Human auditory cortex; Magnetoencephalography; Auditory evoked magnetic field; Steady-state field

\section{Introduction}

Responses evoked by stimuli which follow each other at sufficiently long interstimulus intervals that the auditory system returns (mostly or completely) to its initial state before the next stimulus occurs are called transient evoked responses (Buchwald and Huang, 1975). If the interstimulus interval is shortened to such an extent that the transient response to one stimulus has not died away before the next stimulus is delivered, the compound response that appears is generally referred to as a steady-state response (SSR, Regan, 1982) or steadystate field (SSF) in magnetic recordings. For a linear system, transient and steady-state descriptions of the system's behaviour are equivalent, and a simple superposition of transient responses with the appropriate time lags should perfectly predict the amplitude

*Corresponding author. Fax: +49 (251) 83-6882:

E-mail: pantev@uni-muenster.de. and form of steady-state responses. In this case, both phenomena may be regarded as alternative versions of the same response. However, the auditory system shows several types of non-linear behaviour, and therefore transient and steady-state field recordings may provide different information about auditory function.

The transient auditory evoked fields (AEFs) consist of several components which are labelled according to their latency: the brain-stem AEFs (latency 1-10 ms, Erne et al., 1987), the middle latency AEFs (latency 10-50 ms, Pelizzone et al., 1987; Makela et al., 1994; Pantev et al., 1995), the gamma-band AEF (20-100 ms, Pantev et al., 1991b), the slow AEF (latency 40-250 ms, Reite et al., 1978; Hari and Makela, 1987; Pantev et al., 1991a) and the sustained field (Hari et al., 1980; Pantev et al., 1994). The cessation of transient stimuli 
longer than $100 \mathrm{~ms}$ causes an off-response that is time locked to the end of the stimulus (Onishi and Davis, 1968; Hari et al., 1987; Pantev et al., 1996). Which components are evoked depends mainly on the stimulus paradigm applied. Auditory steady-state responses can be driven by different types of periodically presented short acoustic stimuli, with a maximum response energy for the SSR at stimulus repetition rates in the range $35-40 \mathrm{~Hz}$ (Galambos et al., 1981). Magnetic steady-state fields (SSFS) have been recorded using amplitude modulated tones (Romani et al., 1982) and trains of clicks as stimuli (Hari et al., 1989).

It has been proposed (Galambos et al., 1981) that the electrical SSR, and presumably its magnetic counterpart the SSF, may result from a superposition of middle latency response components $(\mathrm{Na}, \mathrm{Pa}, \mathrm{Nb}$, $\mathrm{Pb}$ ) that occur between 20 and $50 \mathrm{~ms}$ following transient stimulation. Three empirical findings support this 'summation hypothesis' of the auditory SSR: (1) Galambos et al. (1981) showed that the SSR amplitude/frequency characteristic peaking at repetition rates near $40 \mathrm{~Hz}$ is consistent with summation of middle latency responses as the source of the SSR. Because middle latency responses display wave periods near $25-30 \mathrm{~ms}$, middle latency responses elicited by successive acoustic pulses presented at 40 $\mathrm{Hz}$ are likely to be in phase with one another and thus to be preserved in the recorded signal, whereas middle latency responses elicited at repetition rates other than $40 \mathrm{~Hz}$ are likely to be out of phase and subject to wave cancellation. (2) Synthetic SSRs constructed by averaging middle latency responses evoked at $10 \mathrm{~Hz}$ stimulus repetition rates have been observed to closely resemble the true $40 \mathrm{~Hz}$ SSR in amplitude and phase (Galambos et al., 1981; Stapells et al., 1984; Hari et al., 1989). (3) Overlapping cortical regions appear to give rise to SSRs and middle latency responses. Using Gaussian tone pulses with carrier frequency of $1 \mathrm{kHz}$, Pantev et al. (1993) found that the estimated sources of the magnetically recorded Pam middle latency response and the $40 \mathrm{~Hz}$ SSF were centred about 0.5 $\mathrm{cm}$ in an antero-medial direction with respect to cortical sources of the familiar $\mathrm{N1m}$ slow AEF. However, SSF sources could not be spatially differentiated from Pam sources, suggesting that the two phenomena may arise from similar cortical generators. Because only the Pam component of middle latency responses is seen consistently between subjects in magnetic recordings (Pelizzone et al., 1987), it would appear to be the most likely middle latency source of the magnetically recorded SSF which is also a consistent between-subject phenomenon (Pantev et al., 1993).

There is, however, one heretofore neglected finding that does not fit with summation of middle latency responses as the mechanism of the $40 \mathrm{~Hz}$ SSR/SSF. Pantev et al. (1995) investigated simultaneously recorded electric and magnetic middle latency $(\mathrm{Na}, \mathrm{Pa}, \mathrm{Nb}, \mathrm{Pb})$ and slow $(\mathrm{N} 1, \mathrm{P} 2)$ auditory evoked responses elicited by short tone bursts of 500 , 1000 , and $4000 \mathrm{~Hz}$ in 12 subjects. In general accordance with earlier findings (Pelizzone et al., 1987; Pantev et al., 1993), the N1m slow AEF and the Pam component of the middle latency response were observed in all subjects at all tone frequencies, whereas the middle latency components $\mathrm{Nam}, \mathrm{Nbm}$, and $\mathrm{Pbm}$ were present for all tone frequencies in only a minority of subjects (Nam was discernible in two subjects, $\mathrm{Nbm}$ in one subject, and $\mathrm{Pbm}$ in three subjects). Pam sources were again found to be situated about $0.5-1 \mathrm{~cm}$ anterior to the region occupied by $\mathrm{N} 1 \mathrm{~m}$ sources. However, a new result was that the locations of Pam and N1m sources differed significantly along the medio-lateral axis depending on carrier frequency. When stimulus frequencies were increased $\mathrm{N} 1 \mathrm{~m}$ sources shifted to deeper locations along the surface of the supratemporal plane (designated herein as 'medial' tendency tonotopy), whereas the Pam demonstrated the opposite result, namely, at higher frequencies Pam sources were located more laterally than at lower frequencies ('lateral' tendency tonotopy). These results imply that if SSFs are comprised of middle latency responses, and only the Pam wave is consistently seen in magnetic recordings, then the cortical sources determined for SSFs should show the lateral tendency tonotopy of the Pam and not the medial tendency tonotopy of the $\mathrm{N} 1 \mathrm{~m}$. This prediction is contradicted by the results of Romani et al. (1982) who examined differences in SSF sources as a function of stimulus frequency in two subjects. In both subjects SSF sources were observed to shift more medially as a function of frequency, and not laterally as would be expected if SSF responses were comprised of overlapping middle latency waves.

The results of Romani et al. (1982) therefore call into question the view that auditory SSFs consist of summated middle latency responses. Their findings also imply that even though SSF and Pam middle latency responses may arise from spatially overlapping regions when a carrier frequency near 1 $\mathrm{kHz}$ is used (Pantev et al., 1993), the cortical sources of these responses should be spatially separable if the carrier frequency of the $40 \mathrm{~Hz}$ steady-state stimulus is varied across a suitable frequency range. In this paper we report a larger study of tonotopy of SSF sources $(\mathrm{N}=17)$ confirming this prediction, including some cases in which maps for the SSF, Pam, and N1m components were available within the same subjects.

\section{Methods}

\subsection{Subjects}

Eight female and nine male subjects between the age of 20 and 30 years with no history of otological or neurological disorders and normal audiological status (air conduction and bone conduction thresholds no 
more than $10 \mathrm{~dB}$ hearing level in the range from 250 to $8000 \mathrm{~Hz}$ ) participated in this study. All subjects were right-handed as determined using the Edinburgh Handedness Questionnaire (Oldfield, 1971). Informed consent was obtained from each subject after the nature of the study was explained to them in accordance with the principles of the Declaration of Helsinki. The subjects were paid for their participation.

\subsection{Stimulation}

Queues of Gaussian tone pulses (repetition rate of $39 \mathrm{~Hz}$, half-value time of $5 \mathrm{~ms}$ and carrier frequencies of $250,500,1000,2000$ and $4000 \mathrm{~Hz}$, respectively) with a length of $200 \mathrm{~s}$ were presented twice at each frequency (test-retest) to the subject's right ear (contralateral to the investigated hemisphere). The order of presentation of the carrier frequencies was randomized between subjects. Following common practice, we refer herein to carrier frequencies modulated at the $39 \mathrm{~Hz}$ repetition rate as ' $40 \mathrm{~Hz}$ ' steady-state stimulation. The intensity of the acoustic stimuli was set to $60 \mathrm{~dB}$ nHL (normal hearing level). The magnetically silent delivery of the stimuli required a special delivery system with speakers (compressor driver type) outside the magnetically shielded room and echo-free tone transmission through $6.3 \mathrm{~m}$ of plastic tubing $(16 \mathrm{~mm}$ inner diameter) to a silicon car piece. This system was able to provide almost linear frequency characteristics in the range between 200 and $4000 \mathrm{~Hz}$ (deviations less than $\pm 4 \mathrm{~dB}$ ).

To ensure the frequency specificity of the applied steady-state stimuli, the acoustic signals were recorded at the silicon ear piece by means of an artificial ear (Bruel and Kjaer model 4152) equipped with a microphone (Bruel and Kjaer model 4153) coupled to a sound level meter (Bruel and Kjaer model 2203). The power spectra of the Gaussian pulses at the different carrier frequencies used in this experiment are presented in Fig. 1. The spectral bandwidth is the same for all tone pulses since the half-value time of the Gaussian-shaped tone pulses was kept constant. Despite the long stimulus transmission system, the power spectra confirmed the frequency specificity of the stimuli used.

In order to reduce possible variations caused by changes in attention and vigilance, subjects watched cartoons during stimulus presentation. This procedure also reduced the frequency of the subject's eye movements and blinks.

\subsection{Data acquisition}

Recordings were carried out in a magnetically shielded room using a 37-channel biomagnetometer (Biomagnetic Technologies). The detection coils of the biomagnetometer were arranged in a circular concave array with a diameter of $144 \mathrm{~mm}$ and a spherical radius of $122 \mathrm{~mm}$. The distance between the centres of two adjacent coils was $22 \mathrm{~mm}$; each coil itself measured $20 \mathrm{~mm}$ in diameter. The sensors were configured as first-order axial gradiometers with a baseline of $50 \mathrm{~mm}$. The spectral density of the intrinsic noise of each channel was between 5 and 7 $\mathrm{fT} /\left(\mathrm{Hz}^{0.5}\right)$ in the frequency range above $1 \mathrm{~Hz}$. The subjects rested in a right lateral position with their head, neck and body supported by a specially fabricated vacuum mattress. A sensor-position indicator system determined the spatial locations of the sensors relative to the head and indicated if head movements occurred during the recordings. No head movements sufficient to require discarding of data were observed in the study. The sensor array was centred over a point about $1.5 \mathrm{~cm}$ superior to the position T3 of the 10-20 system for electrode placement and was positioned as near as possible to the subject's head. Using a bandwidth from 1 to 100 $\mathrm{Hz}$ and a sampling frequency of $1041.7 \mathrm{~Hz}$ (16-bit ADC), $200 \mathrm{~s}$ of continuous data were recorded twice at each carrier frequency and stored for further analysis.

The procedures used in this study were approved by the Ethics Commission of the Medical Faculty of the University of Muenster and the MEG measurements were classified as completely noninvasive (permission of the Ethics Commission obtained on Nov. 15, 1993).

\subsection{Data analysis}

Epochs contaminated by muscle or eye blink artifacts (signal variations of more than $3 \mathrm{pT}$ in the MEG) were automatically rejected from the averaging procedure. In order to avoid windowing effects of the digital filter, each recorded $200 \mathrm{~s}$ epoch of MEG activity was first band-pass filtered $(24-48 \mathrm{~Hz}$, second-order zero-phase shift Butterworth filter, 12 $\mathrm{dB} / \mathrm{oct}$ ) and then windows of $100 \mathrm{~ms}$ were averaged about 2000 times. Since there were no substantial differences between the test-retest responses, they were cross-averaged. The averaged waveforms obtained for each carrier frequency formed the basis for all further evaluations.

Isofield contour plots were calculated for each sample point. Source analysis based on a single moving equivalent current dipole (ECD) model in a spherical volume conductor (Sarvas, 1987) was applied to the field distributions. The size and the radius of the sphere were determined by fitting a spherical surface to the scalp region underneath the 37 measurement coils using a least squares algorithm. The relative angulation of the pickup coils and the influence of volume currents were taken into account in the source analysis. Source locations, their confidence volumes, and corresponding dipole moments were estimated for each sample point in a 

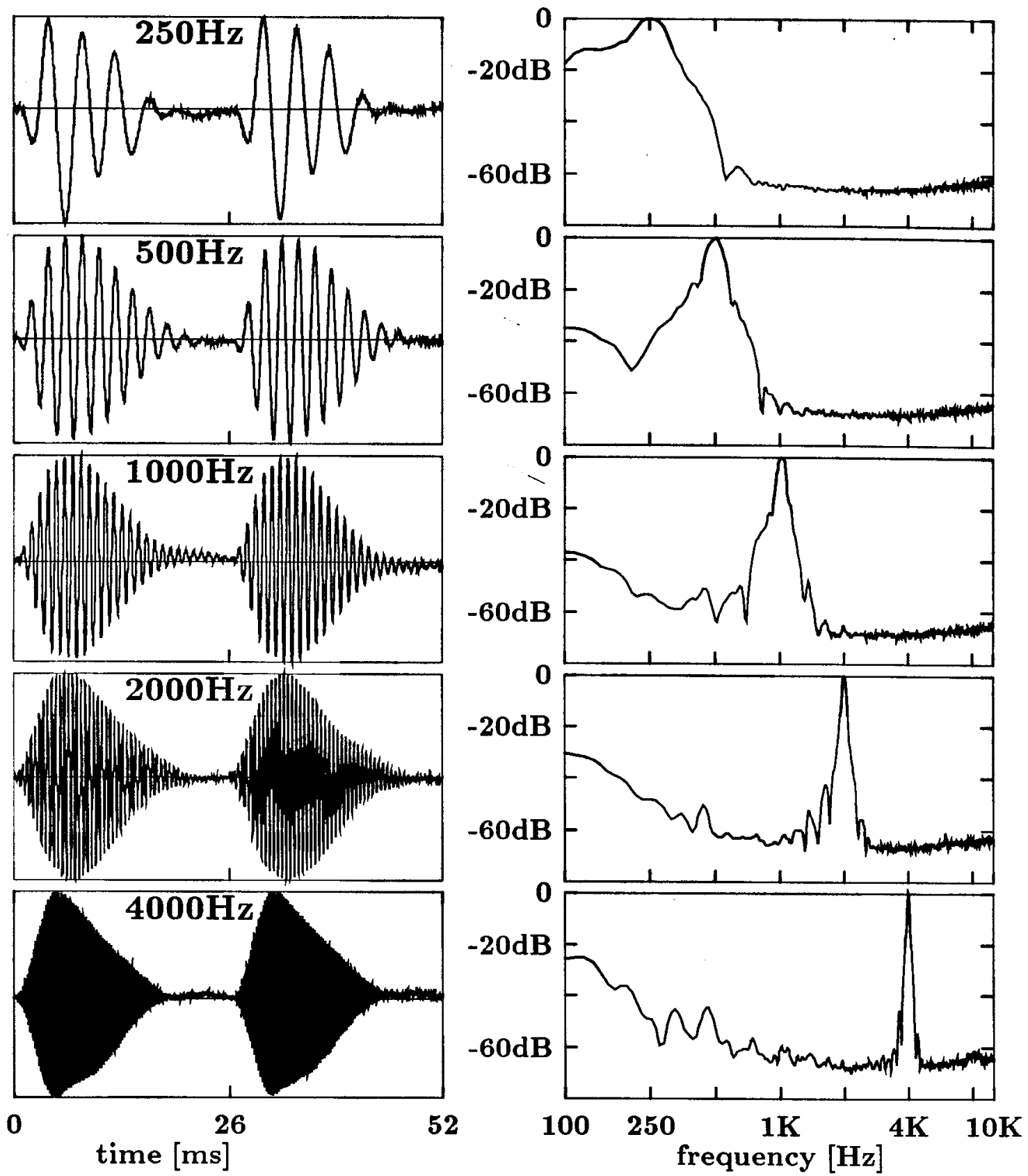

Fig. 1. Left panel: real time description of the steady-state stimulus (two pulses) at each carrier frequency. Right panel: Fourier spectrum of the stimulus at each carrier frequency.

head-based coordinate system. The origin of this coordinate system was set at the midpoint of the mediolateral axis (y-axis) which joined the centre points of the entrance to the acoustic meatii of the left and the right ears (positive towards the left ear). The posteroanterior axis ( $\mathrm{x}$-axis) was oriented from the origin to the nasion (positive towards the nasion) and the inferior- superior axis (z-axis) was perpendicular to the $x-y$ plane (positive towards the vertex).

The estimated locations ( $\mathrm{x}, \mathrm{y}, \mathrm{z}$ coordinates) of the
SSF sources for the different carrier frequencies were determined using median values of the $\mathrm{x}, \mathrm{y}, \mathrm{z}$ coordinates within the $100 \mathrm{~ms}$ response window that achieved the highest 3\% goodness of fit. Only those $x$, $\mathrm{y}$ and $\mathrm{z}$ values were included which met the following additional requirements based on anatomical and statistical considerations: (i) distance of the ECD to the midsagittal plane more than $1.5 \mathrm{~cm}$; (ii) inferiorsuperior value greater than $4 \mathrm{~cm}$; (iii) confidence volume of the source location in each data point smaller than $1 \mathrm{~cm}^{3}$. 

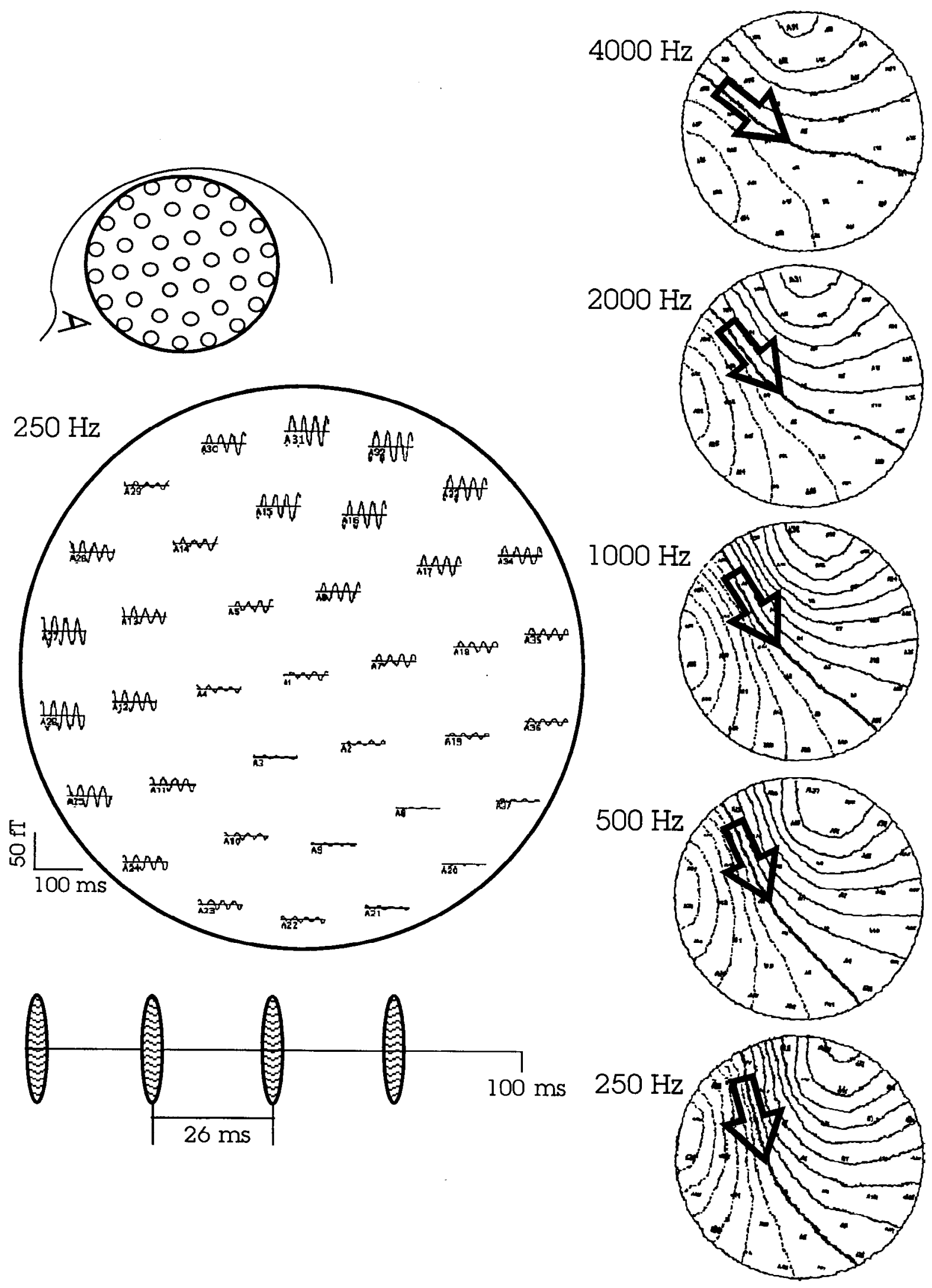

Fig. 2. Left side: magnetic data (time window $100 \mathrm{~ms}$ ) recorded from 37 sensors arranged as shown in the inset (upper left part of the figure). Subject MC, carrier frequency of $250 \mathrm{~Hz}$. An expanded time scale depicting four steady-state tone pulses is shown at the bottom left. Right side: isofield maps at each carrier frequency, for the rms peak closest to $50 \mathrm{~ms}$ in the $100 \mathrm{~ms}$ interval. The arrow depicts the orientation of a single equivalent current dipole fitted to these fields. 

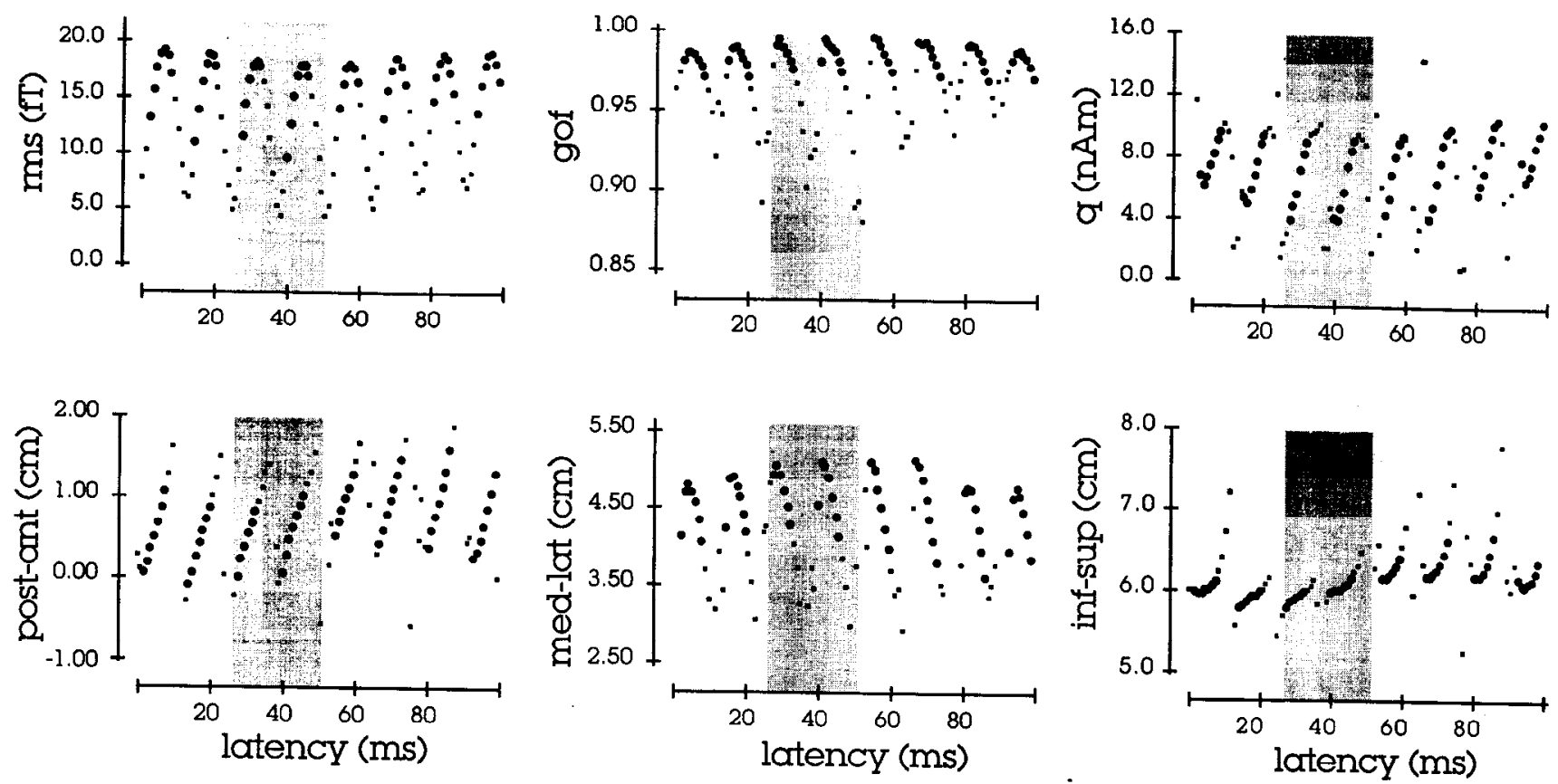

Fig. 3. Source analysis for the data of Fig. $2(250 \mathrm{~Hz})$. Top row, left to right: rms, goodness of fit, and dipole moment. Bottom row, left to right: $3 \mathrm{D}$ source coordinates in the postero-anterior $(\mathrm{x})$, medio-lateral $(\mathrm{y})$, and inferior-superior $(\mathrm{z})$ directions, respectively. The shaded areas represent a time span of $25 \mathrm{~ms}$ synchronized to the occurrence of the second tone pulse $25 \mathrm{~ms}$ into the $100 \mathrm{~ms}$ epoch. Thick dots designate data points meeting the criteria set out in Section 2.

\subsection{MRI overlays}

The source locations calculated for each carrier frequency were superimposed on the corresponding individual MRIs in several cases where we were allowed to obtain the MRI of the subjects. The 3D-MR data sets were generated with a $1.5 \mathrm{~T}$ clinical MR imaging system (Magnetom SP, Siemens). A 3D-T1-weighted gradient- echo sequence was used (TR $10 \mathrm{~ms}$, TE 4 $\mathrm{ms}$, TI $300 \mathrm{~ms}, 128$ partitions, TA 6:48 min) which allowed for a good contrast between grey and white cerebral matter. Using a recording matrix of $256 \times 256$ pixels, a field of view of $250 \mathrm{~mm}$, and a $192 \mathrm{~mm}$ thickness of the sagittally oriented 3D slab, this MPRAGE sequence provided a resolution of $1 \times 1 \mathrm{~mm}$ in the sagittal and $1 \times 1.5 \mathrm{~mm}$ in the reconstructed axial and coronal planes. The anatomical landmarks used to create the MEG head-based 3D-coordinate system (nasion and entrance of the auditory meatus of the left and right ear) were visualized in the MR images by affixing to these points capsules (height and inner diameter of $3 \mathrm{~mm}$ ) filled with Gd-DTPA solution (Schering AG, Berlin) whose short T1 relaxation time provided a high signal in T1-weighted images. The common MEG and MRI anatomical landmarks allowed easy transformation of the head-based Cartesian coordinate system employed by the MEG source analysis to that of the MRI. The MEG source locations were converted into pixels and slice values using the MRI transformation matrix and inserted onto the corresponding MRIs.

\section{Results}

The steady-state field waveforms (averaged over about 4000 epochs of $100 \mathrm{~ms}$ length) for the $250 \mathrm{~Hz}$ carrier frequency of subject MC are shown in Fig. 2 (left side). Each waveform is positioned to correspond to the sensor layout which is superimposed on a sketch of the head in the upper portion of the figure to give an impression of the area of measurement. The time scale of the waveforms (100 ms) corresponds to the enlarged scale shown at the bottom of the figure where four Gaussian tone pulses are illustrated.

Inspection of the waveforms in Fig. 2 shows that the recorded SSF had a clear dipolar pattern with a pronounced field minimum and maximum. The dipolar topography is particularly discernible in the isofield contour maps given in the right side of the figure which show the field distribution obtained at each carrier frequency $(250,500,1000,2000$ and $4000 \mathrm{~Hz}$ ) for the wave maximum closest to $50 \mathrm{~ms}$. The five isofield contour plots are drawn with the same increment of $5 \mathrm{fT}$ with solid lines indicating the outgoing field and dotted lines indicating the ingoing field. The highest field amplitude was observed for the $250 \mathrm{~Hz}$ carrier frequency (75 fT) 

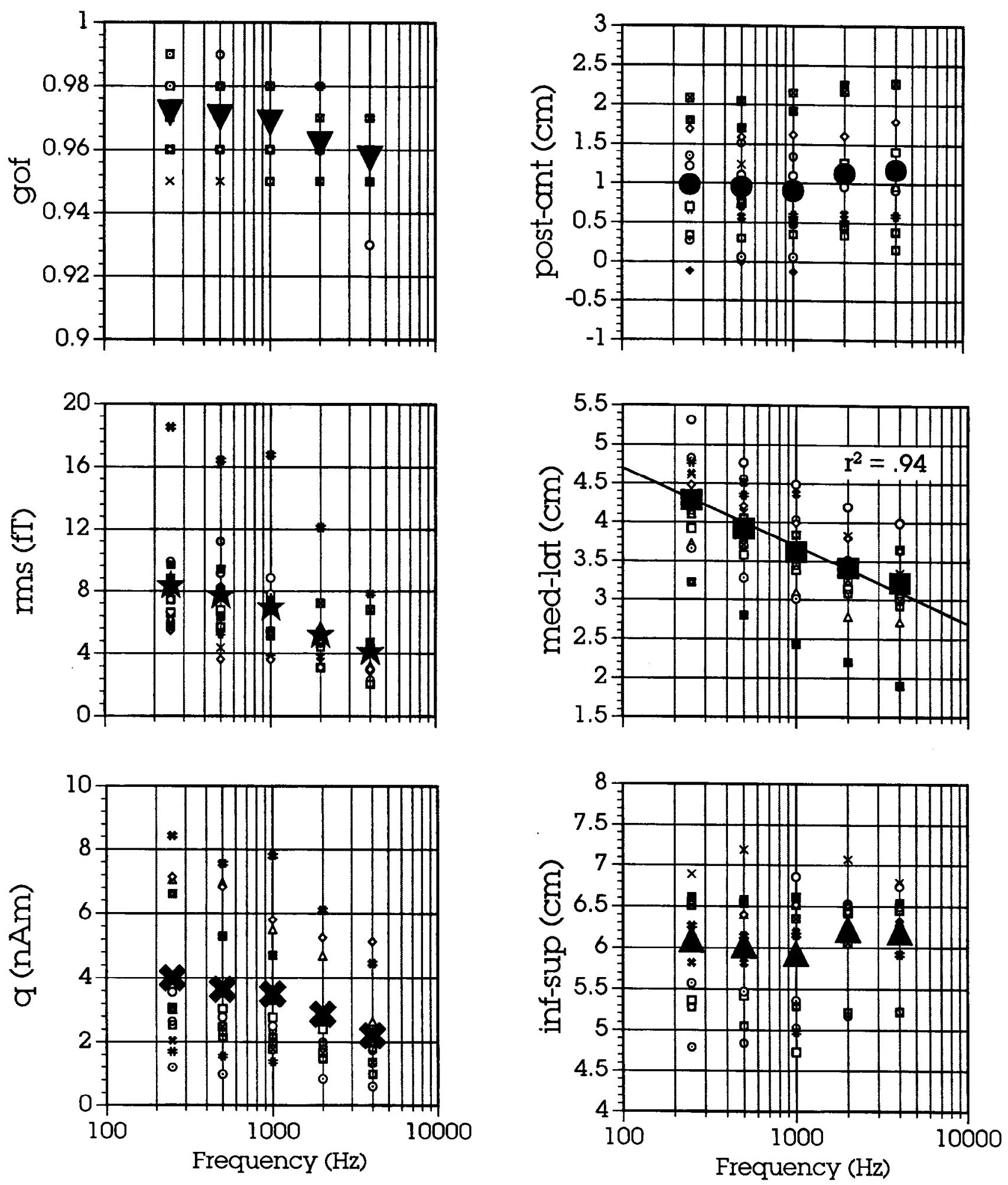

Fig. 4. Dipole parameters for all subjects (small symbols) and grand averages (large symbols). Each small symbol designates a different subject. A least-squares regression line has been fitted to source parameters in the medio-lateral dimension.

and the lowest ( $35 \mathrm{fT}$ ) for $4000 \mathrm{~Hz}$. The projection of the probable equivalent current source is indicated by an arrow placed along the zero isofield contour line in each map. Visual inspection shows a clear rotation of the projection axis by about $40^{\prime}$ counterclockwise as the stimulus carrier frequency changes from $250 \mathrm{~Hz}$ to 4000 

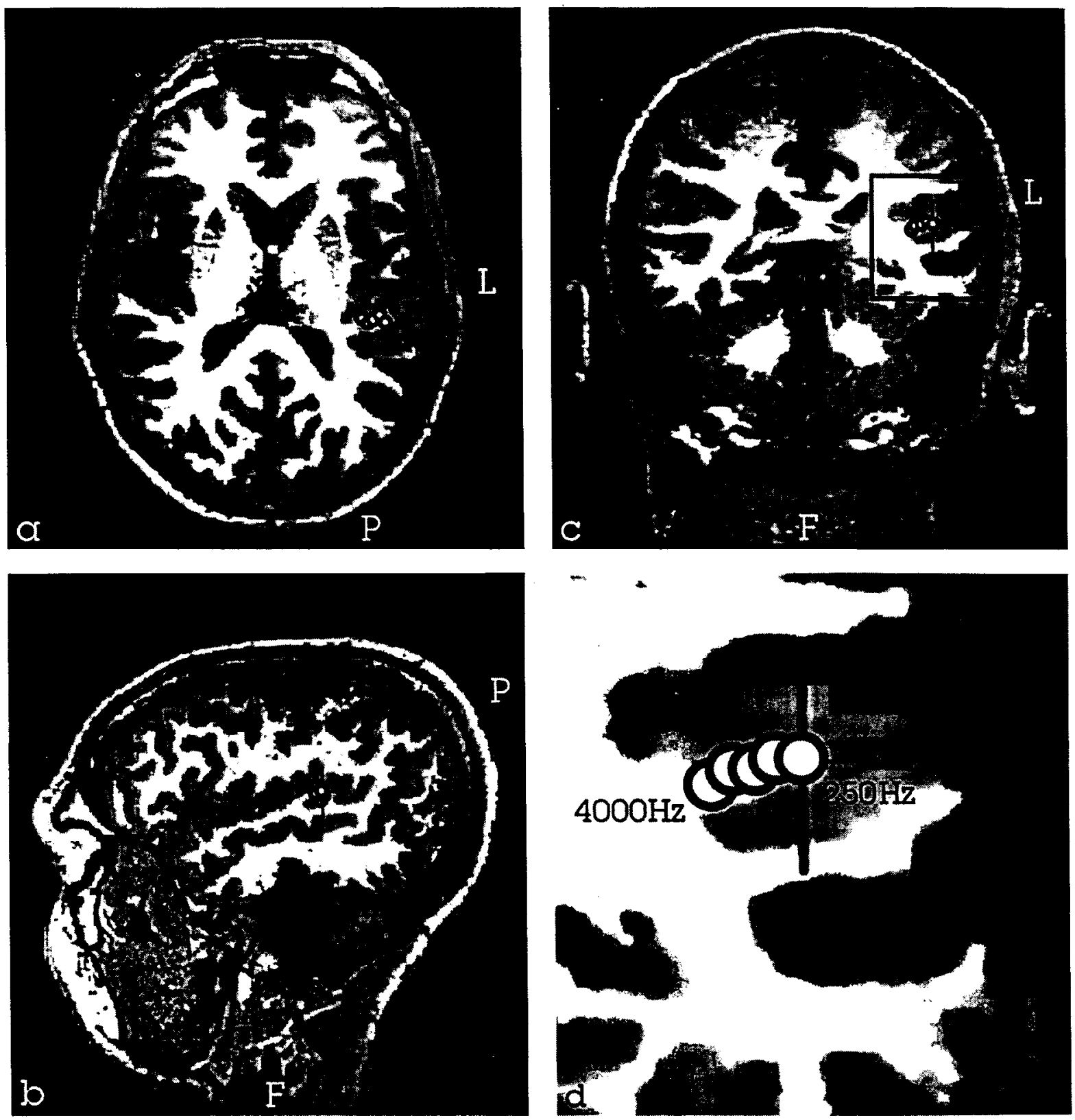

Fig. 5. Superimposition of equivalent current sources computed for subject MC on his MRI. Panels a, b, and c show the axial, coronal, and sagittal planes respectively. Panel $\mathrm{d}$ is an enlargement of the area blocked out in panel c.

Hz. This observation was made for all subjects. The changing amplitude and orientation of the equivalent current source as stimulus frequency was increased suggests that different generator sites were active for the different carrier frequencies.

Fig. 3 displays the source analysis results (rms values, goodness of fit, dipole moment and the 3Dspace coordinates of the estimated equivalent current dipole) for the data set shown on the left side of Fig. 2 (carrier frequency of $250 \mathrm{~Hz}$ ). The shadowed areas represent a time span of $25 \mathrm{~ms}$ synchronized to the occurrence of the second tone pulse at $25 \mathrm{~ms}$ into the $100 \mathrm{~ms}$ epoch. The thick dots shown in the figure designate data points for which at least $97 \%$ of the field variance was explained by a single ECD model and for which confidence volume did not exceed $1 \mathrm{~cm}^{3}$ (cf. Section 2). Due to the field distribution two rms peaks (upper left panel) are seen within the $25 \mathrm{~ms}$ time window related to the appearance of a tone pulse in the $40 \mathrm{~Hz}$ train. The first peak corresponded to an anterior outgoing and the second peak to an anterior ingoing field. The source coordinates (especially postero-anterior and medio-lateral) given in the lower portion of the figure revealed an orderly and progressive shift in successive data points within these rms peaks. This pattern was observed in 15 of our 17 subjects. In the two remaining subjects a 

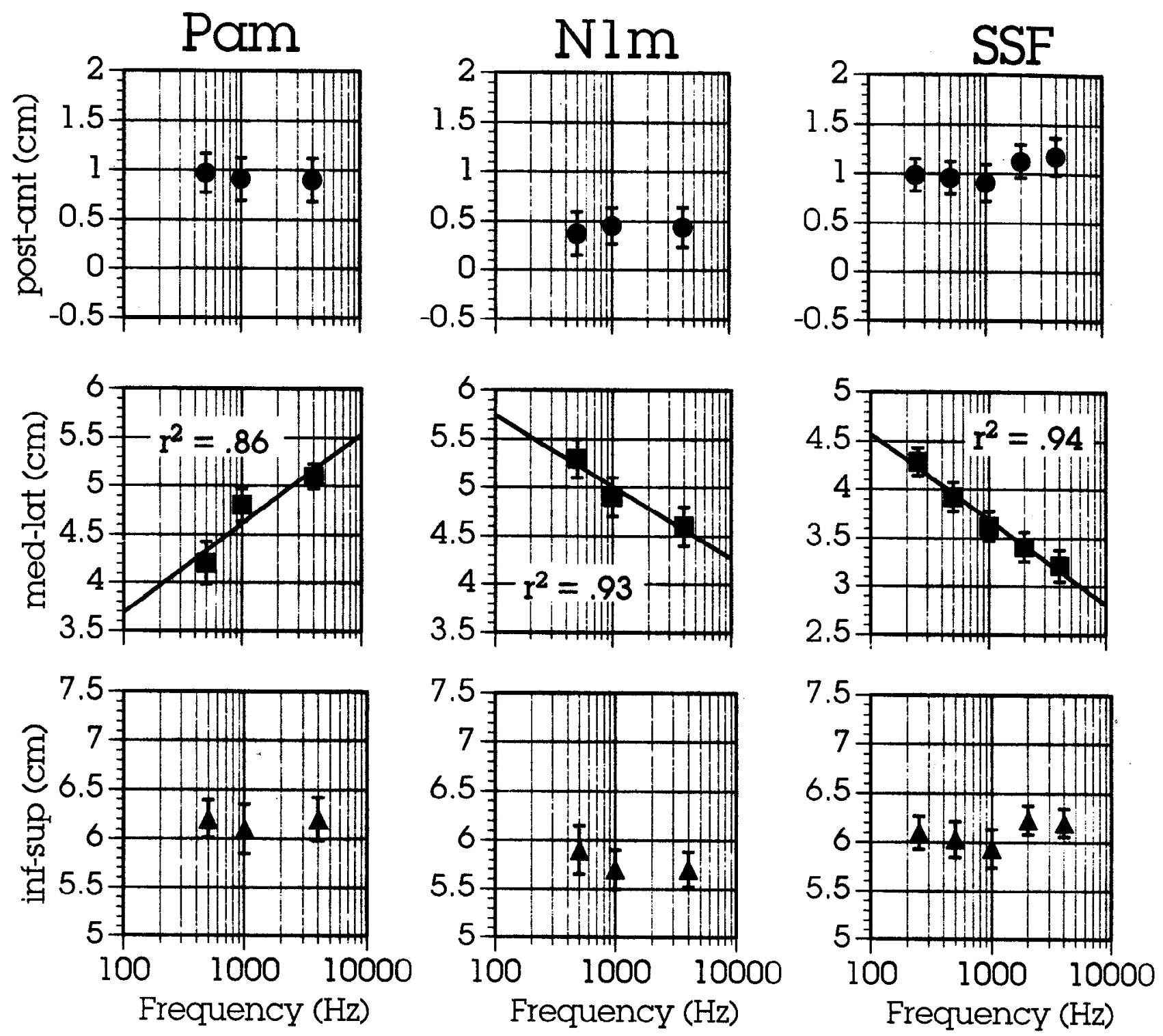

Fig. 6. Left and middle panels: tonotopic maps for Pam and N1m AEFs, respectively, at carrier frequencies of 500, 1000, and 4000 Hz. Right panel: tonotopic map based on SSFs of the present study at carrier frequencies of 250, 500, 1000, 2000, and $4000 \mathrm{~Hz}$ (the grand averages of Fig. 4). A least-squares regression line has been fitted to source parameters in the medio-lateral dimension in each panel. Standard deviations are also shown.

single ECD was not able to explain the data. Although the goodness of fit values were relatively high in these two cases $(>95 \%$ and $>93 \%)$, the calculated cortical sources shifted abruptly between two or more cortical locations depending on which time points were considered. These two subjects were therefore excluded from further analyses.

Dipole parameters based on the criteria set out in Section 2 were determined for each of the 15 subjects and are shown in Fig. 4 (small symbols). Grand averages computed across these subjects are also displayed (large symbols). Inspection of the upper left panel of Fig. 4 shows that goodness of fit typically exceeded $96 \%$ at most carrier frequencies. Goodness of fit also tended to decrease at the higher frequencies, probably as a consequence of the signal-to-noise ratio which also decreased due to diminution of the rms field values (middle left panel, Fig. 4). The fact that rms field values decreased in parallel with mean dipole moment (bottom left panel, Fig. 4) indicates that the equivalent sources corresponding to the higher stimulus frequencies were located deeper in the cortex. This effect is described more directly in the centre right panel of the figure where it is seen that medio-lateral source coordinates computed for each carrier frequency decreased by about $1 \mathrm{~cm}$ as frequency increased over four octaves. A smaller medio-lateral coordinate indicated that the cortical source was situated more deeply, i.e., closer to 
the midsagittal plane of the brain. A shift to deeper sources at higher carrier frequencies was evident in the group data as well as in the data of each individual subject. Overall, source depth shifted medially as a function of carrier frequency (squared correlation coefficient $r^{2}=0.94$ ). Changes in source coordinates in the postero-anterior and inferior-superior directions were smaller and more variable than in the mediolateral direction. In the postero-anterior direction source coordinates shifted about $4 \mathrm{~mm}$ anteriorly at higher stimulus frequencies, whereas in the inferiorsuperior dimension no consistent change could be seen.

For several subjects, estimated SSF source locations were superimposed onto the corresponding sections of their individual MRIs. Fig. 5 gives the results for subject MC whose waveforms and maps were presented previously in Fig. 2. These data demonstrate good agreement of the reconstructed sources with the anatomic structures of the auditory cortex which are shown in axial, coronal, and sagittal views. The SSF source locations were situated in the region of the supratemporal plane and can be preferentially assigned to primary auditory cortical areas (Brodmann's areas 41 and 42). An enlarged representation (Fig. 5d) of the brain area marked in the coronal view (Fig. 5c) shows that the calculated sources of the different frequencies were located in the supratemporal gyrus with sources of higher frequencies located deeper than sources of lower frequencies.

A final analysis compared source parameters determined for the SSF in the present investigation with earlier findings reported by Pantey et al. (1995) for the slow AEF (N1im) and Pam middle latency responses. Fig. 6 depicts the source for the Pam (left panel) and $\mathrm{N} 1 \mathrm{~m}$ (middle panel) responses that were elicited by transient tone stimuli of 500, 1000, and $4000 \mathrm{~Hz}^{1}$. The most notable feature of these data (aside from the previously reported anterior location of Pam sources with respect to N1m sources) is the opposing 'lateral' and 'medial' tonotopy of the Pam and $\mathrm{N} 1 \mathrm{~m}$ waveforms, respectively. For purposes of comparison the SSF maps determined in the present study are reproduced in the right-hand panel of Fig. 6.

Like Pam sources, we found SSF sources to be situated about $0.5 \mathrm{~cm}$ anterior to cortical sources for the N1m of the AEF. SSF sources were also about $1 \mathrm{~cm}$ deeper than $\mathrm{N} 1 \mathrm{~m}$ sources at each carrier

\footnotetext{
1 Short tone bursts of $50 \mathrm{~ms}$ duration $(3 \mathrm{~ms}$ rise and decay time, cosine function) were presented to the subject's right ear (contralateral to the MEG-investigated hemisphere) with an interstimulus interval randomized between 600 and $800 \mathrm{~ms}$. The carrier frequencies were 500, 1000 and 4000 $\mathrm{Hz}$. Blocks of 500 stimuli for each frequency were presented three times in random order.
}

frequency. Close inspection of the figure shows that source parameters for the Pam and SSF responses were similar in each of the three dimensions when a $500 \mathrm{~Hz}$ tone was used to elicit these responses. However, Pam and SSF sources diverged in the medio-lateral dimension when stimulus frequency was varied, with the SSF showing the medial tonotopy of N1m sources in contrast to the lateral tonotopy of Pam sources. Three subjects for whom the tonotopy of the SSF sources was determined in the present study also participated in the previous study (Pantev et al., 1995) in which the tonotopy of Pam and N1m sources was determined. The individual results of these subjects matched closely the group results presented in Fig. 6 .

\section{Discussion}

Source analyses reported by Pantey et al. (1995) found that neural generators for the Pam middle latency AEF evoked by transient tone pulses were centred near the surface of the supratemporal gyrus, and that these generators shifted laterally with logarithmic increases in the carrier frequency of the eliciting stimulus ('lateral' tendency tonotopy). Cortical generators for the N1m AEF, on the other hand, were found to be posterior and inferior to those of the Pam and to shift medially with the logarithm of the stimulus frequency in the same subjects ('medial' tendency tonotopy). The present experiment compared the cortical sources of the $40 \mathrm{~Hz}$ auditory SSF with cortical sources of the Pam and $\mathrm{N} 1 \mathrm{~m}$ at carrier frequencies between 500 and $4000 \mathrm{~Hz}$. The SSF sources observed at these frequencies were found to display a medial tonotopy resembling the $\mathrm{N} 1 \mathrm{~m}$, opposite to the lateral tonotopy of sources of the middle latency Pam (cf. Fig. 6). Medial tendency SSF tonotopy was observed in each of our subjects $(\mathrm{N}=15)$ with a very high percentage of the variance accounted for by the source analysis, including three subjects for whom Pam and N1m maps were also available.

These findings call into question the hypothesis that the $40 \mathrm{~Hz} \mathrm{SSF}$ is comprised of summated transient middle latency responses evoked by auditory steady-state stimuli. Because their tonotopy is opposite, the neural sources of the SSF and Pam responses are clearly differentiable. Furthermore, it is unlikely that the middle latency components $\mathrm{Nam}, \mathrm{Nbm}$, or $\mathrm{Pbm}$ were responsible for the SSFs we measured here. Previous observations have shown that these components recorded magnetically are present in fewer than one quarter of the subjects at the tone frequencies used in our investigation (Pantev et al., 1995; see Pelizzone et al., 1987 and Makela et al., 1994, for congruent findings). However, all subjects showed a detectable SSF 
in the present study, which implies that neural sources distinct from $\mathrm{Nam}, \mathrm{Nbm}$, and $\mathrm{Pbm}$ sources must have generated these SSFs. It should be noted that the observed dissociation of SSF and middle latency sources does not depend on whether the single ECD model that we used to describe the data was biologically precise. Even if multiple generators were present, the extracted parameters nevertheless described components that should have come out identically for the SSF and Pam if the former was merely a linear superimposition of the latter.

Our source analysis also provided information regarding the relation of SSF sources to sources for the N1m slow AEF and other transient responses of the auditory system. SSF sources were found to he centred about $1 \mathrm{~cm}$ medially and $0.5 \mathrm{~cm}$ anteriorly with respect to sources of the $\mathrm{N} 1 \mathrm{~m}$. Hence the cortical networks that supported these responses were not fully overlapping despite their similar tonotopy. The anterior location of SSF sources relative to those for the N1m agrees with the early report of Makela and Hari (1987) who elicited the SSF with $500 \mathrm{~ms}$ trains of clicks. A further response of the auditory system from which the SSF appears dissociable is the transient gamma band response (GBR; Pantev et al., 1991b). Although gamma-band and steady-state responses have similar wave periods near $25 \mathrm{~ms}$, the GBR does not show tonotopic order (Bertrand and Pantev, 1994; Pantev and Elbert, 1994). Furthermore, source determinations for the magnetically recorded GBF suggest that this response arises from cortical activity centred between the N1m and SSF (Pantev et al., 1993). These differences in source parameters and tonotopic organization of the sources suggest that GBR and SSF responses are separable in terms of their neural mechanisms and functional roles (Forss et al., 1993; Pantev et al., 1993).

The coordinates that we obtained for SSF sources and subsequent superimposition of these sources on the MRIs of several subjects were consistent with neural activity centred in primary auditory cortical regions of the supratemporal gyrus (Brodmann's areas 41 and 42). Recent animal studies in which intracortical (Karmos et al., 1993) and subdural field potentials (Franowicz and Barth, 1995) were recorded are consistent with our results in suggesting primary auditory cortex as the source of the $40 \mathrm{~Hz}$ SSR, and also in distinguishing the $40 \mathrm{~Hz}$ SSR from transient middle latency responses. Karmos et al. (1993) recorded local field potentials from indwelling electrodes placed at different levels of the auditory cortex in the cat. Transient stimulation consisting of a single $0.1 \mathrm{~ms}$ click evoked several short latency (11-60 ms) evoked potentials that reversed in polarity with cortical depth, whereas continuous $40 \mathrm{~Hz}$ click stimulation produced a different local field pattern that did not invert across the cortical layers that were sampled. Karmos et al. (1993) concluded that the field patterns evoked by continuous $40 \mathrm{~Hz}$ stimulation could not be explained by summation of transient responses evoked by single clicks. Franowicz and Barth (1995) recorded from an array of subdural electrodes placed on the surface of the auditory cortex of rats and found that the $40 \mathrm{~Hz}$ SSR and early (P1a/N1a) and late (P1b/N1b) middle latency responses could be adequately modelled by a small number of spatio-temporal components. Isopotential maps showed that the spatial distribution of the early P1a/N1a middle latency response (14 and $26 \mathrm{~ms}$, respectively) was centred in primary auditory cortex (area 41) whereas the spatial distribution of the later P1b/N1b middle latency response (16 and $28 \mathrm{~ms}$ respectively) was focused in secondary auditory cortex (areas 20 and 36). Isopotential maps for the 40 $\mathrm{Hz}$ SSR were also centred over area 41 (primary auditory cortex) as were isopotential maps for the early $\mathrm{P} 1 \mathrm{a} / \mathrm{N} 1 \mathrm{a}$ middle latency response, but the spatial distribution of the $40 \mathrm{~Hz}$ SSR was not concentric with that of the early P1a/N1a middle latency complex which typically had a wider spatial distribution than that of the SSR. Franowicz and Barth (1995) concluded that parallel and asynchronous activation of distinct thalamocortical projections was responsible for generating the early and late middle latency response complexes, and that the neuronal sources responsible for the $40 \mathrm{~Hz}$ SSR overlapped with only a small subset of the sources of these middle latency complexes and were directly related to none of their temporal constituents ${ }^{2}$. Our finding of opposite tonotopy between middle latency and SSF responses in humans confirms the separability of these responses as suggested by the results of Franowicz and Barth (1995).

The SSF consists of field reversals that occur at the repetition rate of the steady-state stimulus (see Fig. 2). This reversal, which is described by the two successive rms peaks following each steady-state stimulus as shown in Fig. 3, did not appear to reflect communication between spatially segregated cortical regions, because the source currents underlying the field reversal were localized to the same cortical area. The simplest interpretation of these findings is that the field reversal reflected intracellular currents of opposite polarity that were consequent on

\footnotetext{
2 Franowicz and Barth (1995) also concluded that neural sources of the 40 $\mathrm{Hz}$ SSR were separable from spontaneous and click-induced gamma band oscillations. Unlike the SSR, spontaneous gamma band oscillations were localized to both primary and secondary auditory cortex whereas transient gamma band responses induced by bursts of $40 \mathrm{~Hz}$ stimulation did not phase-lock to the stimulus as the $40 \mathrm{~Hz}$ response did.
} 
depolarization and repolarization of the same cortical neurones activated by each steady-state pulse. With regard to the question of mechanisms, it should be noted that the source locations of the SSF changed systematically by almost $1 \mathrm{~cm}$ in the sagittal $(\mathrm{x}-\mathrm{z})$ and coronal $(y-z)$ planes over the time of $10 \mathrm{~ms}$ for each of eight peaks as shown in Fig. 3. The same observation was made by Hari et al. (1989) in one of their two subjects for whom source analysis was carried out. Although the trajectory of the equivalent current dipole occurred within a cortical surface of about 1 $\mathrm{cm}^{3}$, which cannot be easily explained by the single equivalent dipole model, this observation has to be considered as significant. It was consistently reproducible during all peaks of the SSF and was found for the five different carrier frequencies in 15 of the 17 subjects. This type of behaviour in source space suggests the existence of two or more closely located dipoles with biphasic dipole moments and phase lags. In the majority of the cases (15 out of 17 ) the activity of these two or more closely located dipoles was successively approximated by the activity of a single ECD. Most likely the failure of the single ECD model in the two cases was based on variations in the individual neuroanatomy, which have changed the constellation of the closely located dipoles and therefore their resulting field pattern could not be explained sufficiently by the single ECD.

Steady-state responses induced by acoustic probes are known to show a pronounced amplitude enhancement when steady-state stimuli are presented at repetition rates between 35 and $40 \mathrm{~Hz}$ (Galambos et al., 1981; Romani et al., 1982; Stapells et al., 1984; Picton et al., 1987; Hari et al., 1989). If summation of middle latency responses is not responsible for this frequency-amplitude characteristic, the question is raised as to its mechanism. One interpretation appeals to the fact that neurones and neural assemblies are nonlinear elements which may show a frequency dependence in their amplitudes. If a nonlinear system is periodically stimulated, harmonics, combination frequencies, and subharmonic components may evolve. Most of all, the system will display a distinct pattern around resonance frequencies for which the system is tuned. The SSR amplitude/frequency characteristic peaking near this modulation rate (Stapells et al., 1984; Regan, 1989) may be just one example of such behaviour and a consequence of ubiquitous non-linearity in the auditory system. However, linear accounts are also possible. For example, a frequency/amplitude characteristic peaking near $40 \mathrm{~Hz}$ could arise from a convergence of afferent and reafferent thalamocortical input to auditory cortical processing areas for which the $25 \mathrm{~ms}$ wave period of the $40 \mathrm{~Hz}$ modulation frequency is optimal. Because afferent and reafferent thalamocortical inputs do not appear to project to the same cortical targets (Winer and Larue, 1987), summation in magnetic or electrical recordings could occur without a concurrent effect on perception which might otherwise be expected to track the SSR frequency/amplitude characteristic. Alternatively, $40 \mathrm{~Hz}$ stimulation may activate neurones in lemniscal pathways that respond at preferred modulation rates (Lins et al., 1995). According to Rees et al. (1986) approximately $80 \%$ of the neurones in the inferior colliculus respond optimally at frequencies between 10 and $120 \mathrm{~Hz}$, and about $50 \%$ between 30 and $100 \mathrm{~Hz}$. Different steady-state stimulus rates may selectively activate specific clusters of neurones in these pathways which receive input from different regions of the cochlea and distribute to the cortex in a tonotopic arrangement (Lins and Picton, 1995)

\section{Acknowledgments}

This research was supported by grants from the Deutsche Forschungsgemeinschaft (Klinische Forschergruppe 'Biomagnetismus und Biosignalanalyse'), the Natural Sciences and Engineering Research Council of Canada (OGP0000132), and the NATO Division of Scientific Affairs (940648). We thank Drs. Riita Hari, Scott Makeig and Jyrki Makela for their helpful comments on an earlier version of the manuscript.

\section{References}

Bertrand, 0. and Pantey, C. (1994) Stimulus frequency dependence of the transient oscillatory auditory evoked responses $(40 \mathrm{~Hz})$ studied by electric and magnetic recordings in human. In: C. Pantev, T. Elbert and B. Lutkenhoner (Eds.), Oscillatory Event Related Brain Dynamics, Plenum, New York, pp. 231-242.

Buchwald, J.S. and Huang, C.M. (1975) Far-field acoustic response: origins in the cat. Science 189, 382-384.

Erne, S.N., Scheer, H.J., Hoke, M., Pantev, C. and Lutkenhoner, B. (1987) Brainstem auditory evoked magnetic fields in response to stimulation with brief tone pulses. Int. J. Neurosci. 37, 115- 125.

Forss, N., Makela, J.P., McEvoy, L. and Hari, R. (1993) Temporal integration and oscillatory responses of the human auditory cortex revealed by evoked magnetic fields to click trains. Hear. Res. 68, 89-96.

Franowicz, M.N. and Barth, D.S. (1995) Comparison of evoked potentials and high frequency (gamma-band) oscillating potentials in rat auditory cortex. J. Neurophysiol. 74, 96-112.

Galambos, R., Makeig, 5. and Talmachoff, P.J. (1981) A 40-Hz audi- tory potential recorded from the human scalp. Proc. Natl. Acad. Sci. (USA) 78, 2643-2647.

Hari, R. and Makela, J. (1987) Neuromagnetic studies of the human auditory cortex. In: C. Barber and T. Blum (Eds.), Evoked Potentials Ill. Butterworth, Boston, MA, pp. 130-135.

Hari, R., Aittoniemi, K., Jarvinen, M.L., Katila, T. and Varpula, T. (1980) Auditory evoked transient and sustained magnetic 
fields of the human brain. Localization of neural generators. Exp. Brain Res. 40, 237-240.

Hari, R., Pelizzone, M., Makela, J.P., Hallstrom, J., Leinonen, L. and Lounasmaa, O.V. (1987) Neuromagnetic responses of the human auditory cortex to on- and offsets of noise bursts. Audiology 26, 31-43.

Hari, R., Hamalainen, M. and Joutsiniemi, S.L. (1989) Neuromagnetic steady-state responses to auditory stimuli. J. Acoust. Soc. Am. 86, 1033-1039.

Karmos, G., Makela, J.P., Ulbert, 1. and Winkler, 1. (1993) Evidence for intracortical generation of the auditory $40-\mathrm{Hz}$ response in cat. In: H.J. Heinze, T.F. Munte and G.R. Mangun (Eds.), New Development in Event-Related Potentials. Birkenhauser, Boston, MA, pp. 87-93.

Lins, O.G. and Picton, T.W. (1995) Auditory steady-state responses to multiple simultaneous stimuli. Electroenceph. Clin. Neurophysiol. 96, 420-432.

Lins, O.G., Picton, P.E., Picton, T.W., Champagne, S.C. and Durieux Smith, A. (1995) Auditory steady-state responses to tones amplitude-modulated at 80-110 Hz. J. Acoust. Soc. Am. 97, 3051-3063.

Makela, J.P. and Hari, R. (1987) Evidence for cortical origin of the $40 \mathrm{~Hz}$ auditory evoked response in man. Electroenceph. Clin. Neurophysiol. 66, 539-546.

Makela, J.P., Hamalainen, M., Hari, R. and McEvoy, L. (1994) Whole-head mapping of middle-latency auditory evoked magnetic fields. Electroenceph. Clin. Neurophysiol. 92, 414421.

Oldfield, R.C. (1971) The assessment and analysis of handedness: the Edinburgh inventory. Neurophysiologia 9, 97-113.

Onishi, 5. and Davis, H. (1968) Effect of duration and rise time of tone bursts on evoked V potentials. J. Acoust. Soc. Am. 44, 582- 591.

Pantev, C. and Elbert, T. (1994) The transient auditory evoked gamma-band field. In: C. Pantey, T. Elbert and B. Lutkenhbner (Eds.), Oscillatory Event Related Brain Dynamics. Plenum, New York, pp. 219-230.

Pantey, C., Gallen, C., Hampson, S., Buchanan, 5. and Sobel, D. (1991a) Reproducibility and validity of neuromagnetic source localization using a large array biomagnetometer. Am. J. EEG Technol. 31, 83-101.

Pantev, C., Makeig, 8., Hoke, M., Galambos, R., Hampson, S. and Gallen, C. (1991b) Human auditory evoked gamma-band magnetic fields. Proc. Nati. Acad. Sci. (USA) 88, 8996-9000.

Pantev, C., Elbert, T., Makeig, S., Hampson, S., Eulitz, C. and Hoke, M. (1993) Relationship of transient and steady-state auditory evoked fields. Electroenceph. Clin. Neurophysiol. 88, 389-396.
Pantey, C., Eulitz, C., Elbert, T. and Hoke, M. (1994) The auditory evoked sustained field: origin and frequency dependence. Electroenceph. Clin. Neurophysiol. 90, 82-90.

Pantev, C., Bertrand, 0., Eulitz, C., Verkindt, C., Hampson, S., Schuirer, G. and Elbert, T. (1995) Specific tonotopic organizations of different areas of the human auditory cortex revealed by simultaneous magnetic and electric recordings. Electroenceph. Clin. Neurophysiol. 94, 26-40.

Pantev, C., Eulitz, C., Hampson, S., Ross, B. and Roberts, L.E. (1996) The auditory evoked 'off response: sources and comparison with the 'on' and the 'sustained' response. Ear Hear. in press.

Pelizzone, M., Hari, R., Makela, J.P., Huttunen, J., Ahlfors, S. and Hamalainen, M. (1987) Cortical origin of middle-latency auditory evoked responses in man. Neurosci. Lett. 82, 303307.

Picton, T.W., Skinner, C.R., Champagne, S.C., Kellett, A.J. and Maiste, A.C. (1987) Potentials evoked by the sinusoidal modulation of the amplitude or frequency of a tone. J. Acoust. Soc. Am. 82, 165--178.

Rees, A., Green, G.R. and Kay, R.H. (1986) Steady-state evoked responses to sinusoidally amplitude-modulated sounds recorded in man. Hear. Res. 23, 123-133.

Regan, D. (1982) Comparison of transient and steady-state methods. Ann. NY Acad. Sci. 45-71.

Regan, D. (1989) Human Brain Electrophysiology: Evoked Potentials and Evoked Magnetic Fields in Science and Medicine. Elsevier, New York.

Reite, M., Edrich, J., Zimmerman, J.T. and Zimmerman, J.E. (1978) Human magnetic auditory evoked fields. Electroonceph. Clin. Neurophysiol. 45, 114-117.

Romani, G.L., Williamson, S.J., Kaufman, L. and Brenner, D. (1982) Characterization of the human auditory cortex by the neuromagnetic method. Exp. Brain Res. 47, 381-393.

Sarvas, J. (1987) Basic mathematical and electromagnetic concepts of the biomagnetic inverse problem. Phys. Med. Biol. $32,11-22$.

Stapells, D.R., Linden, D., Suffield, J.B., Hamel, G. and Picton, T.W. (1984) Human auditory steady state potentials. Ear Hear. $5,105-113$.

Winer, J.A. and Larue, D.T. (1987) Patterns of reciprocity in auditory thalamocortical and corticothalarnic connects: study with horseradish peroxidase and autoradiographic methods in the rat medial geniculate body. J. Comp. Neurol. 257, 282315 . 